\title{
Die tierische Besiedlung des hyporheischen Interstitials eines Urgebirgsbaches unter dem Einfluß von allochthoner Nährstoffzufuhr
}

\author{
von \\ Hans-Gerd PIEPER*
}

\begin{abstract}
SUMMARY
The animal population of the hyporheic interstitial in a primary rock stream under the influence of the supply of allochthonous organic matter.

The Mettma, a mountain stream in the Black Forest, W. Germany, was investigated for effects of input of nutrients and energy by domestic waste water and effluent from a brewery introduced at one specific point. The investigation time ranged from May 1970 to February 1971. Initially, there is an impoverishment and structural changes of the biocoenosis in the hyporheic interstitial. There is an almost complete $\mathrm{O}_{2}$-depletion immediately after the waste water has been added. In winter, temperatures in the interstitial were higher than those in the current. No direct correlation between population densities and amount of organic matter were observed where allochthonous nutrients had been added. Population densites of multicellular animals were from 0 to $1.2 \times 10^{5}$ per $0.1 \mathrm{~m}^{3}$ of sediment. $4.1 \mathrm{~km}$ further downstream the fauna is similar to that above the waste water inlet, while at $7.35 \mathrm{~km}$ downstream of it is slightly less dense.
\end{abstract}

\section{EINLEITUNG}

Das hyporheische Interstitial ist der Grenzbiotop zwischen dem oberirdischen Ökosystem des Fließgewässers und dem subterranen Ökosystem des Grundwassers. (Chappuis, 1943; Angelier, 1953; Husmann, 1956, 1966; Orghidan, 1959; Schwoerbel, 1961, 1961a, 1967; Tilzer, 1968).

Zwischen der Nährstoffracht der fließenden Welle und dem Sediment der Stromsohle bestehen wichtige nährstoffregulierende Beziehungen, wie Schwoerbel (1972) bei Untersuchungen über den Phosphataustausch Sediment-Wasser zeigen konnte. Für die Untersuchung des Einflusses der Nährstoffracht der fließenden Welle auf die Biozönose des hyporheischen Interstitials erscheint die Selbstreinigungsstrecke eines mit Abwässern belasteten Baches besonders geeignet.

* Limnologisches Institut der Albert-Ludwigs Universität Freiburg und Limnologische Flußstation des Max-Planck-Instituts für Limnologie D 6407 Schlitz, BRD. 
Ziel der vorliegenden Arbeit war es, zu klären, welchen Veränderungen die Biozönose des hyporheischen Interstitials im Verlauf der Selbstreinigung des Baches unterworfen ist.

\section{DAS UNTERSUCHUNGSGEWÄSSER}

\subsection{GEOGRAPHISCHE LAGE}

Die Mettma entspringt unweit vom südöstlichen Ende des Schlucksees im Urgestein. Die Untersuchungsstecke beginnt unterhalb der Ortschaft RothausBrünlisbach und erstreckt sich über die Länge von 7,5 km. Diese Fließstrecke

\section{Mettma, Lage der Probenstellen}

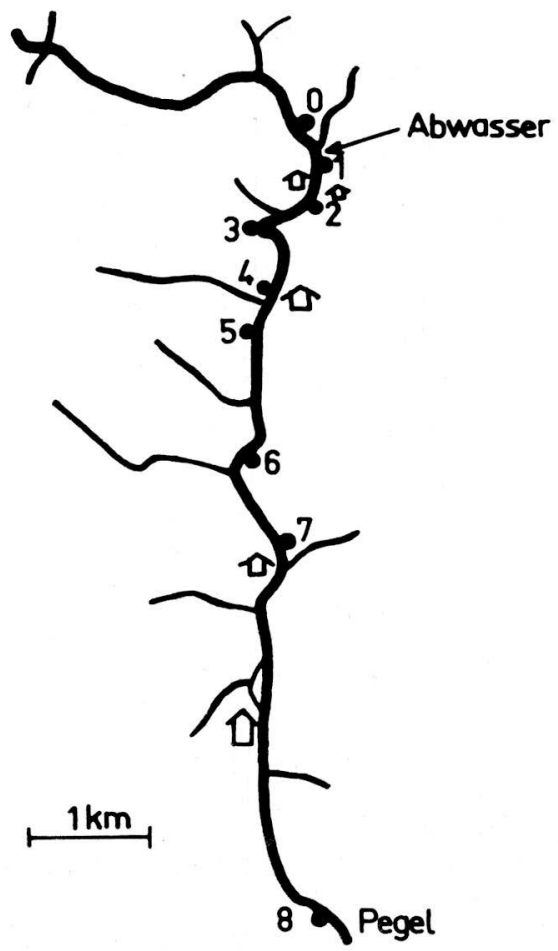

Abb. 1. Im Text werden die Probenstellen mit P0, Pl usw. bezeichnet. 
wurde in 9 Untersuchungsstellen eingeteilt (Abb. 1). Die Probenstelle 0 liegt $884 \mathrm{~m}$ ü. NN, die letzte Probenstelle, an der sich ein Pegel befindet, liegt $730 \mathrm{~m}$ ü NN. Das durchschnittliche Gefälle des untersuchten Bachabschnittes beträgt $2,2 \%$.

Zwischen Probenstelle 0 und Probenstelle 1 befindet sich eine punktförmige Abwassereinleitungsstelle.

\subsection{DAS ABWASSER}

Das Abwasser setzt sich aus häuslichen Abwässern und zum größten Teil aus Brauereiabwässern der Rothausbrauerei zusammen. Die Abwässer der Brauerei mit Mälzerei besitzen einen hohen Gehalt an leicht abbaubaren Substanzen. Sie enthalten insbesondere gelöste Zucker, löslich gemachte Eiweißstoffe und anorganische Salze. Das Abwasser wird mechanisch geklärt in den Brünlisbach entlassen; dieser mündet kurz nach der Kläranlage in die Mettma.

\subsection{LAGE DER PROBESTELLEN (Abb. 1)}

Die Lage der Probenstellen richtet sich nach dieser Einleitungsstelle. $200 \mathrm{~m}$ oberhalb dieser Einleitungsstelle liegt die 0-Stelle, deren Wasserqualität und deren qualitative und quantitative Besiedlung zum Vergleich mit jedem untersuchten Punkt der folgenden Selbstreinigungsstrecke herangezogen wird.

\section{METHODIK}

Zur Ermittlung der quantitativen und qualitativen Besiedlung der Sedimente wurden organismenund detritusfreie Sandproben in das hyporheische Interstitial eingegraben. Plastikröhren von 160 $\mathrm{cm}^{3}$ Inhalt wurden mit passenden Schraubdeckeln versehen. Die Kappe der Schraubdeckel wurde ausgeschnitten und die Öffnung mit Nylongaze von $1 \mathrm{~mm}$ Maschenweite bespannt.

Die für die Exposition verwendeten Sande wurden dem Fließgewässer entnommen, sorgfältig ausgewaschen, getrocknt und mit Prüfsieben DIN 4188 in die Korngrößenfraktionen 0,5-1 mm, 1-2 mm, 2-4 mm zerlegt. Die Röhren wurden mit Sediment jeweils einer Korngrößenfraktion gefüllt und mit den vorbereiteten Deckeln versehen. Durch die $1 \mathrm{~mm}$ weiten Öffnungen der Gaze können die meisten Fließwasser- und Interstitialbewohner beliebig passieren; natürlich muß für alle Korngrößen die gleiche Maschenweite verwendet werden.

Die zur Exposition vorbereiteten Sandröhren wurden entsprechend der Korngröße des eingegüllten Sediments mit einer farbigen Banderole markiert, die gewährleistet, daß auch in getrübtem Wasser die Korngröße sofort sicher erkannt und die richtige Röhre eingeholt werden kann. Die Röhren wurden an den bezeichneten Probenstellen in etwa gleicher Entfernung von der freien Wasserlinie, soweit das möglich war, in gleicher Tiefe und Lage zum Gewässer im Sediment exponie: $\mathrm{t}$ (in 20-30 cm Tiefe und $20 \mathrm{~cm}$ von der freien Wasserlinie; horizontal im Bachgrund und senkrecht zur Fließrichtung). Es wurden jeweils fünf Röhren mit jeder Korngrößenfraktion an einer Probenstelle eingegraben. Die nach Ablauf der Expositionszeit eingeholten Röhren wurden entleert und das Sediment vorsichtig ausgeschlämmt. Der Detritus wurde in Nylongaze Nr. 25 mit $60 \mu$ Maschenweite aufgefangen, in eine unterseits linierte Petrischale gegeben und unter dem Binokular quantitativ und qualitativ auf Organismen ausgezähit. Der Detritus einschließlich des durch die Gaze hindurchgegangenen Anteils wurde in einen Glastiegel überführt und 48 Stunden lang 
bei $100^{\circ} \mathrm{C}$ im Trockenschrank getrocknet. Der organische Anteil wurde durch Veraschen im Muffelofen bei $600^{\circ} \mathrm{C}$ nach 2 Stunden bęstimmt.

Das zur chemischen Untersuchung,benötigte Porenwasser wurde mit Hilfe von einem Schlagrohr (Messing) gewonnen, das oberhalb der Spitze Bohrungen aufweist. Ich verwendete $120 \mathrm{~cm}$ lange Messingrohre mit einer massiven Messingspitze und 10 Bohrungen als Eintrittsöffnungen für das Porenwasser.

Mit ebenso gewonnenem Porenwasser wurde der $\mathrm{CO}_{2}$-Gehalt (Feldmethode $\mathrm{n} / 20 \mathrm{NaOH}$ ) und der Säuregrad (Merck-Indikator) bestimmt (Fehlergrenze $\star 0,5)$.

Die Temperatur wurde mit einem Stabtermometer in $20-30 \mathrm{~cm}$ Tiefe unmittelbar an den Expositionsstellen gemessen.

\section{ERGEBNISSE}

\subsection{MILIEUFAKTOREN}

Die $\mathrm{O}_{2}$-Verhältnisse

Die Sauerstoffkurven im Längsprofil zeigten zunächst einen starken Abfall der $\mathrm{O}_{2}$-Sättigung des Porenwassers kurz nach der Abwassereinleitungsstelle an P1 (Abb. 2). Im Verlauf der Selbstreinigungsstrecke des Baches stiegen die Sauerstoffsättigungswerte langsam an und erreichten an P7 nach ca. $4 \mathrm{~km}$ Fließstrecke wieder ähnliche Werte wie an der Bezugsprobenstelle P0. Vergleiche

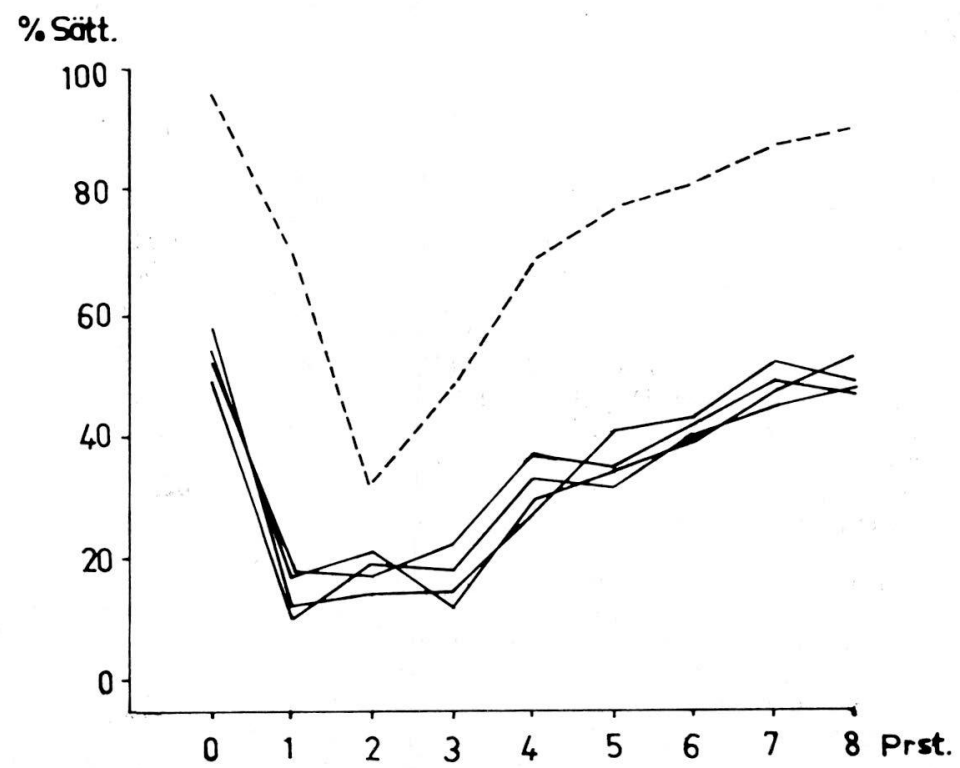

Abb. 2. $\mathrm{O}_{2}$-Sättigung des Interstitialwassers zu verschiedenen Zeiten im Längsprofil. Gestrichelte Kurve: $\mathrm{O}_{2}$-Sättigungswerte des epigäischen Bachwassers (nach FRANKE, 1972). 
der $\mathrm{O}_{2}$-Kurven des hyporheischen Interstitials mit den $\mathrm{O}_{2}$-Kurven der fließenden Welle (Franke, 1972) zeigten Divergenzen. In der fließenden Welle trat ein erhebliches Sauerstoffdefizit bezeichnender Weise erst an P2 ein.

Freie $\mathrm{CO}_{2}$

Die Konzentration der freien Kohlensäure verlief im Interstitial der des Sauerstoffs entgegengesetzt. Im Verlauf der Selbstreinigungsstrecke sank der $\mathrm{CO}_{2}-$ Gehalt relativ stark wieder ab und erreichte nach Abschluß der Selbstreinigung geringfügig höhere Werte als vor der Abwassereinleitungsstelle. (Abb. 3).

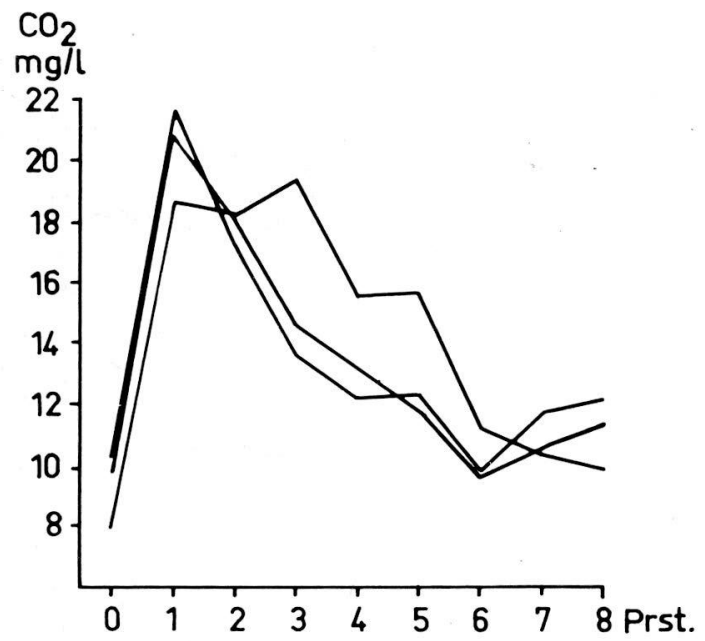

Abb. 3. $\mathrm{CO}_{2}-$ Gehalt (mg/l) im Längsprofil.

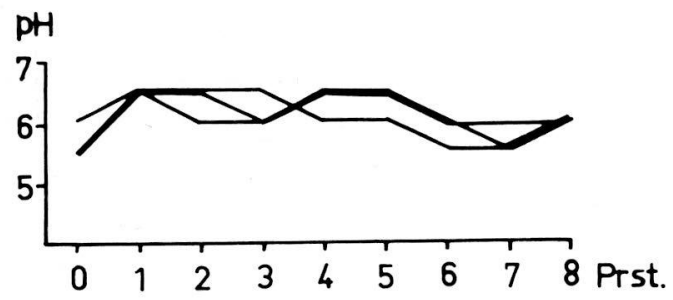

Abb. 4. Der pH-Wert des hyporheischen Interstitials im Längsprofil zu verschiedenen Zeiten.

Der pH-Wert

Auf der gesamten Untersuchungsstrecke lag der $\mathrm{pH}$-Wert im Interstitial im Bereich von 5,5-6,5 (Abb. 4). In der fliessenden Welle erreichte das $\mathrm{pH}$ Werte von 6,2-7,25 (Franke, 1972). 


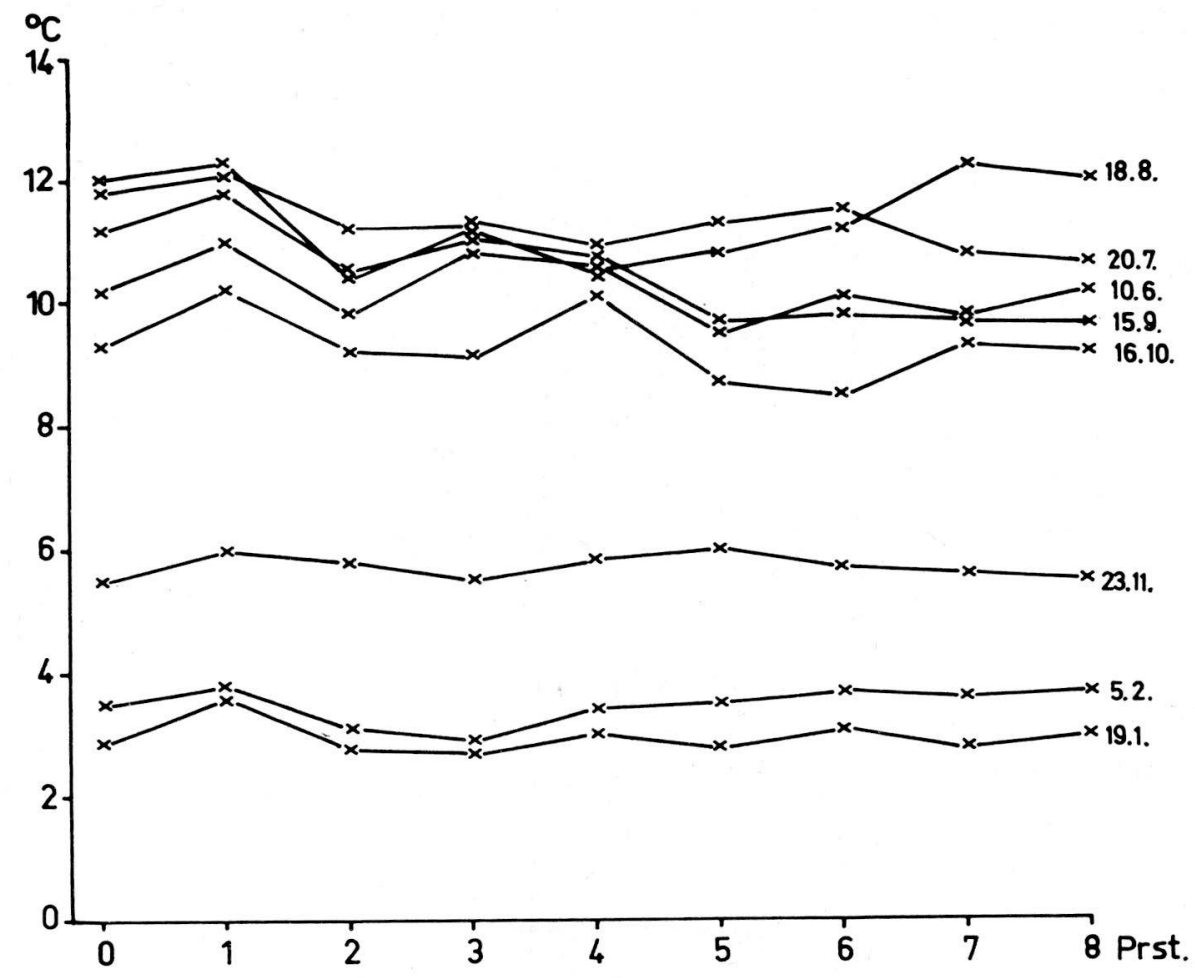

Abb. 5. Temperaturverhältnisse im Interstitial. 


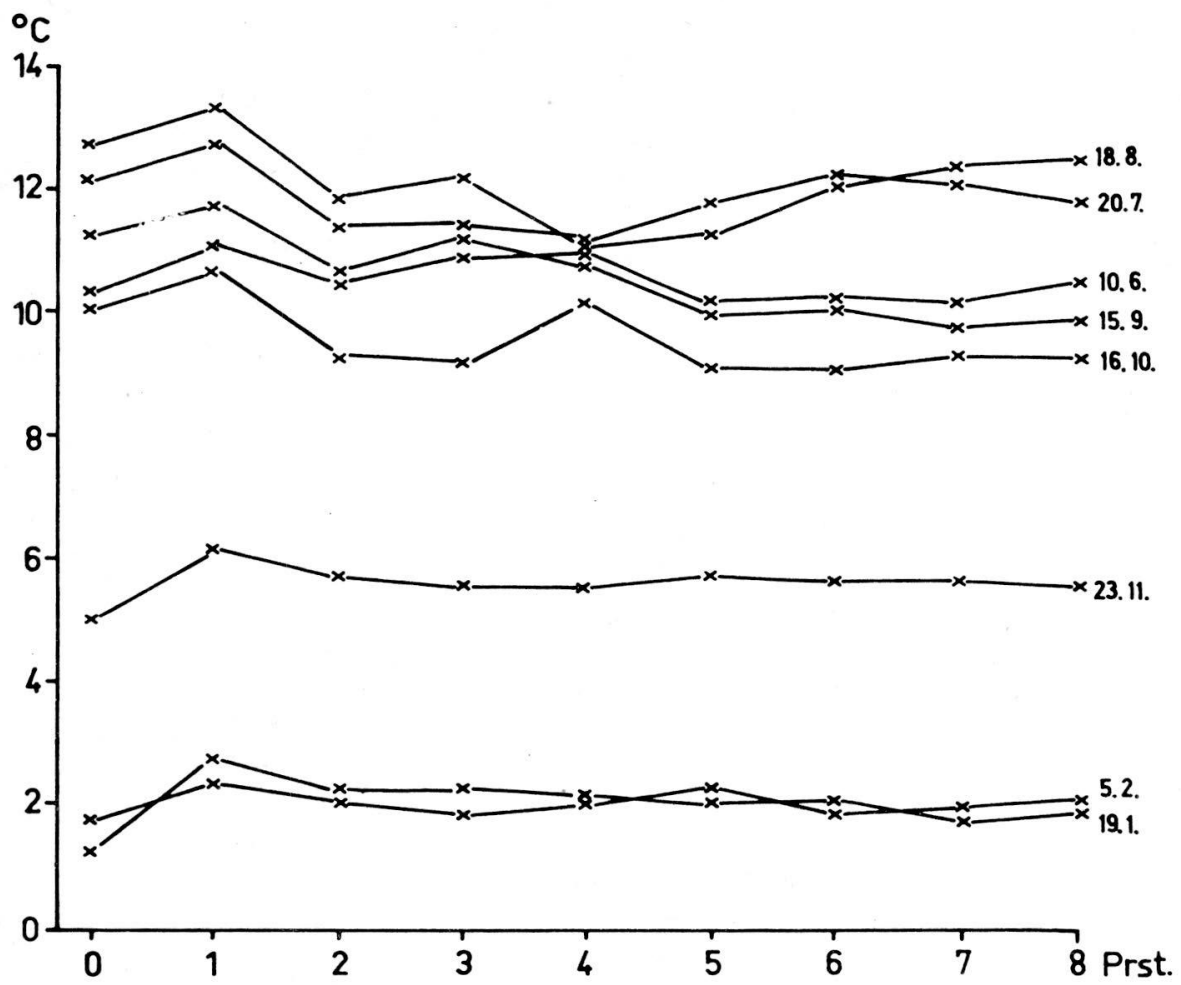

Abb. 6. Temperaturverhältnisse im epigäischen Bachwasser.

Temperaturverhältnisse

Die Temperaturverhältnisse im hyporheischen Interstitial waren denen des epigäischen Bachwassers in etwas gemilderter Form gleich. Häufig lagen die absoluten Werte im Sommer und im Herbst unter denen des Oberflächenwassers (Abb. 5 und 6). In den Wintermonaten Januar und Februar lagen die Temperaturen im hyporheischen Interstitial durchweg höher als in der fliessenden Welle. Die Temperaturdifferenz zwischen epigäischen Bachwasser und dem Interstitial betrug im Winter maximal $1,6^{\circ} \mathrm{C}$. 

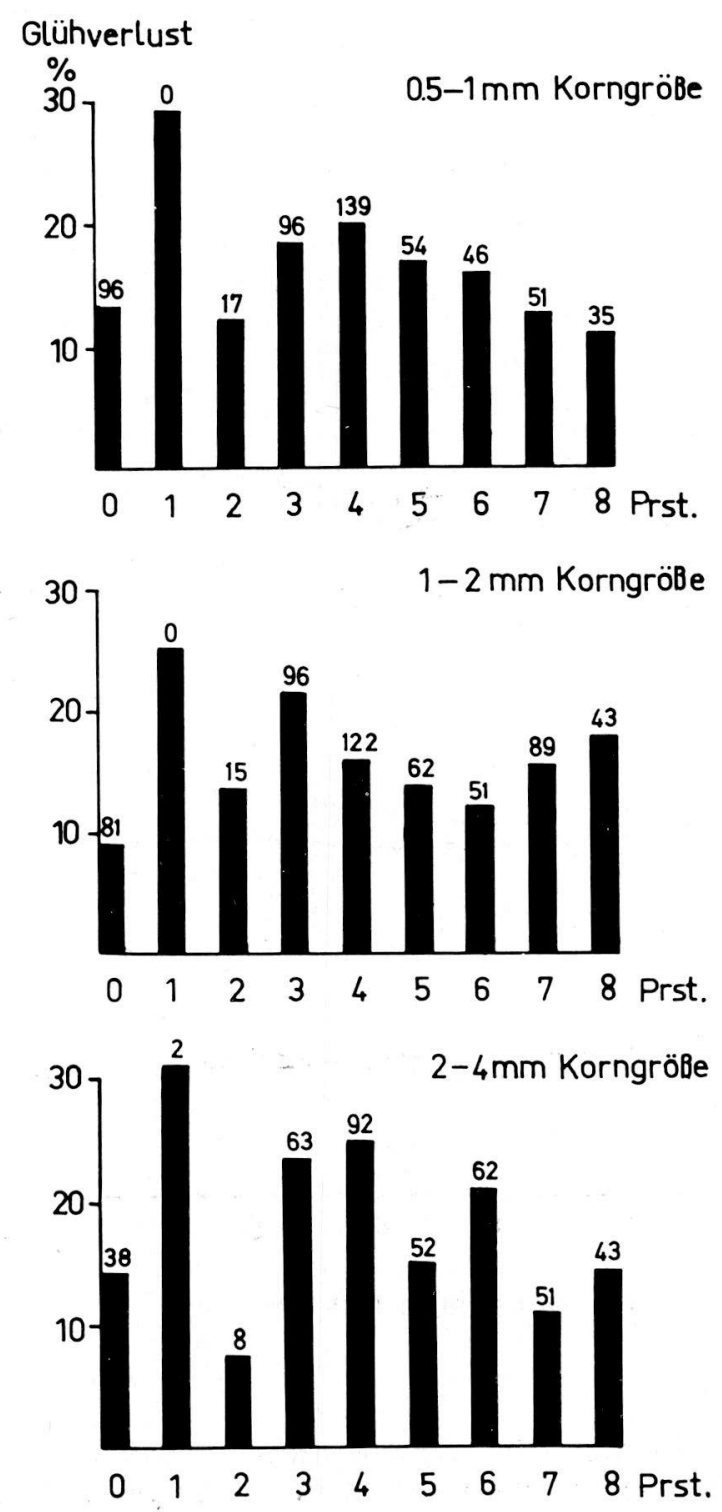

Abb. 7. Prozentualer Anteil der organischen Substanz am Detritus bei verschiedenen Korngrößen an den einzelnen Probestellen. Die Zahl über der Säule gibt die absolute Anzahl der gefundenen Organismen pro $160 \mathrm{~cm}^{3}$ Sediment an. 
Die organische Substanz (Abb. 7)

Der prozentuale Anteil der organischen Substanz am Gesamtdetritus war gegenüber der P0 and P3, P4, P5, P6 deutlich erhöht, an P2, P7 und P8 unterschiedlich. Unter Berücksichtigung der Korngröße der exponierten Sande zeigten sich große Schwankungen in den Werten für die organische Substanz an den einzelnen Probenstellen. (Resultate für die Menge an Gesamtdetritus hier nicht veröffentlicht).

\subsection{DIE TIERISCHE BESIEDLUNG}

In der vorliegenden Arbeit habe ich mich darauf beschränkt, Untersuchungen über den Anteil der einzelnen Organismengruppen an der tierischen Gesamtbesiedlung des hyporheischen Interstitials vorzunehmen. Es wurden drei Untersuchungsserien im Zeitraum vom 22.7.1970 bis zum 13.10.1970 durchgeführt. Ich beschränke mich auf die Veröffentlichungen der dritten Serie. Die Expositionszeit betrug jeweils 20 Tage.

Folgende Tiergruppen, die sowohl im freien Fließgewässer als auch im hyporheischen Interstitial vorkommen, konnten festgestellt werden: Oligochaeten, Nematoden, Turbellarien, Cyclopiden, Harpacticiden, Ostracoden, Amphipoden, Hydrachnellen, Hydrachnellennymphen und Rotatorien. Arten mit ausschließlich hypogäischer Verbreitung gehören ebenfalls den genannten Gruppen an. Gruppen, deren Entwicklungsstadien teilweise im hyporheischen Biotop leben, sind folgende: Ephemeriden, Plecopteren, Chironomiden, andere Insekten und Coelenteraten ( Hydra). Besonders häufig auftretende Formen wurden genauer erfasst, so daß sich folgende Unterteilung ergibt, die ich bei meinen Untersuchungen verwendet habe und auf die ich mich weiterhin beziehen werde: Tubificiden und Tubificidenkokons (es handelt sich ausschließlich um Tubifex tubifex), Naididen (Naiden und Chaetogaster), Lumbriculiden, Nematoden, Ostracoden, Rotatorien, Plecopterenlarven und -larvulae, Ephemeridenlarven und -larvulae, Chironomidenlarven, sonstige Insektenlarven, Turbellarien, Cyclopiden, Amphipoden, Harpacticiden, Hydrachnellen und Hydrachnellennymphen, Coelenteraten. Aus der Gesamtheit der Biozönose wurden Bakterien, "niedere Pilze" und Protozoen bei den Untersuchungen nicht berücksichtigt (vgl. dazu Reichardt, 1972).

Einen Einblick in die Änderung der tierischen Besiedlung im Verlauf der Selbstreinigungstrecke vermitteln die Abbildungen 8 und 8a.

Die Oligochaeten, vor allem Tubifex tubifex, haben an der Gesamtbiozönose einen bedeutenden Anteil. An P0 überwiegen die Naididen gegenüber Tubifex. Lumbriculiden wurden nur an P4 und P5 gefunden. Nach P5 tritt T. tubirex nur noch sporadisch auf.

Die Naididen meiden die Probenstellen (P1, P2, P3). An P4 fand ich Naiden, an P5 Chaetogaster in geringer Anzahl. Die Naididen haben dann bis einschließlich P8 einen nicht unbedeutenden Anteil an der Biozönose.

Die Nematoden waren an allen Probenstellen, mit Ausnahme von P1, stets zu finden. 
Turbellarien fehlten völlig an P1, P2, P3 und P4, erst an P6 haben sie einen bedeutenderen Anteil an der Biozönose.

Die Cyclopiden und Harpacticiden fehlten an P1, P2, P3 und P4 völlig. Hohe Anteile zeigten sie an der Vergleichsprobenstelle 0 und an P7 und P8.

Ostracoden traten in einigen Proben mit hohen prozentualen Anteilen an der Besiedlung an P6 auf, während ihr Anteil an der Besiedlung an P8 sich im Mittel dem von P0 anglich.

Amphipoden wurden nur an P8 gefunden.

Hydrachnellen und Hydrachnellennymphen traten an P0, P7 und P8 auf.

Rotatorien zeigten ein sporadisches Auftreten (es ist möglich, daß beim Auszählen Rotatorien übersehen oder beim Ausschlämmen der Proben zerquetscht wurden).

Unter den Insektenlarven dominierten die Chironomidenlarven. Sie traten an allen Probenstellen, ausser P1 und P2, auf.

Die Ephemeridenlarven und Ephemeridenlarvulae stellten in einigen Proben prozentual den größeren Anteil an der Besiedlung als die Plecopterenlarven und Plecopterenlarvulae, durchgehend waren Plecopterenlarven häufiger zu finden. An P1, P2, P3, P4 fehlten beide Gruppen völlig.

Die unter sonstigen Insektenlarven zusammengefassten Larven fehlten an P2 und P3 völlig. Ein geringes Vorkommen dieser Gruppe fand sich an P4, im überigen traten sie mit unterschiedlicher Häufigkeit an allen übrigen Probenstellen auf.

Hydra wurde nur an P0 und P7 gefunden.

\subsection{DIE BESIEDLUNGSDICHTE}

Die Verwendung von Sandröhren ermöglicht eine quantitativ exakte Angabe der Tierzahlen pro Sedimentmenge. Die Anzahl der Organismen wurde auf $0,1 \mathrm{~m}^{3}$ berechnet. Der Umrechnungsfaktor beträgt 625 bezogen auf $160 \mathrm{~cm}^{3}$.

Die Besiedlungsdichte an den einzelnen Probenstellen (Korngrößenfraktionen jeweils zusammengefasst) zeigt für die Bezugsprobenstelle 0 eine Besiedlungsdichte von ca. 33300 Individuen pro 0,1 $\mathrm{m}^{3}$ (Abb. 9). Diese Besiedlungsdichte wurde annähernd an P8 mit 31400 Individuen pro $0,1 \mathrm{~m}^{3}$ erreicht, während an P3, P4, P5, P6, P7 höhere Besiedlungsdichten zu verzeichnen waren. Das Besiedlungsmaximum lag an P4 mit ca. 121600 Individuen pro $0,1 \mathrm{~m}^{3}$. Das bedeutete eine Besetzung von 1,2 Tieren pro $\mathrm{cm}^{3}$ Sediment, denen aber nur $40 \%$ des Sedimentvolumens zur Verfügung standen. An Pl ist überhaupt keine Besiedlung festzustellen und an P2 eine sehr geringe.

\section{DISKUSSION}

Die Veränderung in der tierischen Besiedlung des hyporheischen Interstitials bei allochthoner Nährstoffzufuhr zeigen deutlich den Einfluß des epigäischen Bachwassers auf diesen Lebensraum. Die Änderungen der Milieubedingungen 
führten zunächst zu einer Verarmung und Umstrukturierung der Biozönose, die sogar bis zur Vernichtung dieser Biozönose durch Sauerstoffmangel führen kann, wie die Verhältnisse an Pl zeigten. Die Wiederbesiedlung erfolgte zunächst durch Organismen, die keine typischen Interstitialbewohner sind, wie Tubificiden, Nematoden und Rotatorien. T. tubifex ist wegen seines Massenvorkommens an den Probenstellen P3, P4, P5 besonders wichtig. Dies dürfte vom äußerst günstigen Nahrungsangebot bestimmt sein. T. tubifex toleriert darüber hinaus ein sehr geringes Sauerstoffangebot. Wachs (1967) wies darauf hin, daß infolge der unaufhörlichen selektiven Aufnahme von tiefergelegenen Substratmengen die Tubificiden eine progressive und zusätzliche Zersetzung des Schlammes schaffen und sie bereiten durch die lockere Lagerung des koprogenen Materials auf der Schlammoberfläche den Weg für den aeroben Abbau des organischen Anteils der Sedimente. Die Tubificiden müssen als wichtige Glieder der biologischen Selbstreinigung angesprochen werden (Liebmann, 1960a), da sie "die zur biologischen Selbstreinigung führenden Prozesse" (Solowiew, 1924) regulieren.

Das Maximum der Tubificidenbesiedlung im hyporheischen Interstitial der Mettma fand sich an P4. Nach der Probenstelle P4 war die Tubificidenbesiedlung rückläufig; die Substrateigenschaften müssen für Tubificiden nach der Probenstelle P4 als nicht mehr optimal bezeichnet werden. Hier zeigten sich auch die ersten Änderungen in der Biozönose, vier weitere Tiergruppen (Lumbriculiden, Naiden, Chaetogaster und sonstige Insektenlarven) treten gegenüber der Probenstelle P3 auf. Es muß aber betont werden, daß der Anteil dieser vier Tiergruppen an der Gesamtbesiedlung äußerst gering ist. Einerseits dürfte sicherlich für dieses Ergebnis die immer noch niedrige $\mathrm{O}_{2}$-Konzentration, die maximal 40\% Sättigung erreichte, eine Rolle spielen. Andererseits zeigt die durch die allochthone Nährstoffzufuhr eingetretene Saprobisierung der Mettma mit Beginn der Probenstelle P4 eine rückläufige Tendenz, wie die Abnahme der Tubificidenbesiedlung nach dieser Probenstelle beweist. Neben der deutlichen Zunahme von Naididen, sonstigen Insektenlarven und Lumbriculiden an der Gesamtbesiedlung der Probenstelle P5 weist das Vorkommen von Cyclopiden, Plecopteren und Turbellarien auf eine neue qualitative Änderung der Lebensbedingungen hin. Dies ist mit Einschränkung der Grund dafür, daß an P6 alle für die Gesamtbesiedlung des hyporheischen Interstitials maßgeblichen Tiergruppen wieder vorhanden sind und daß die Besiedlungsdichte ein außerordentlich hohes Maß erreicht.

Typisch für den Vorgang der Wiederbesiedlung ist, daß jede der Tiergruppen zunächst mit nur wenigen Individuen auftritt, an der nächstfolgenden Probenstelle jedoch zahlreich vertreten ist. Ein dichteres Zusammenlegen der Probenstellen hätte mehr Aufschluß darüber erbracht, nach wieviel km Fließstrecke die einzelnen Tiergruppen das Intestitial erneut besiedeln. Ungeklärt bleibt allerdings hier die Frage, woher die Wiederbesiedlung des hyporheischen Interstitials durch die dort lebenden Tiere erfolgt. Grundsätzlich gibt es für die ausschließlich im hyporheischen Interstitial lebenden Tiere zwei Möglichkeiten für die Wiederbesiedlung: Die horizontale Einwanderung durch das Lückensystem des Interstitials oder die Vertikalwanderung von tiefergelegenen 

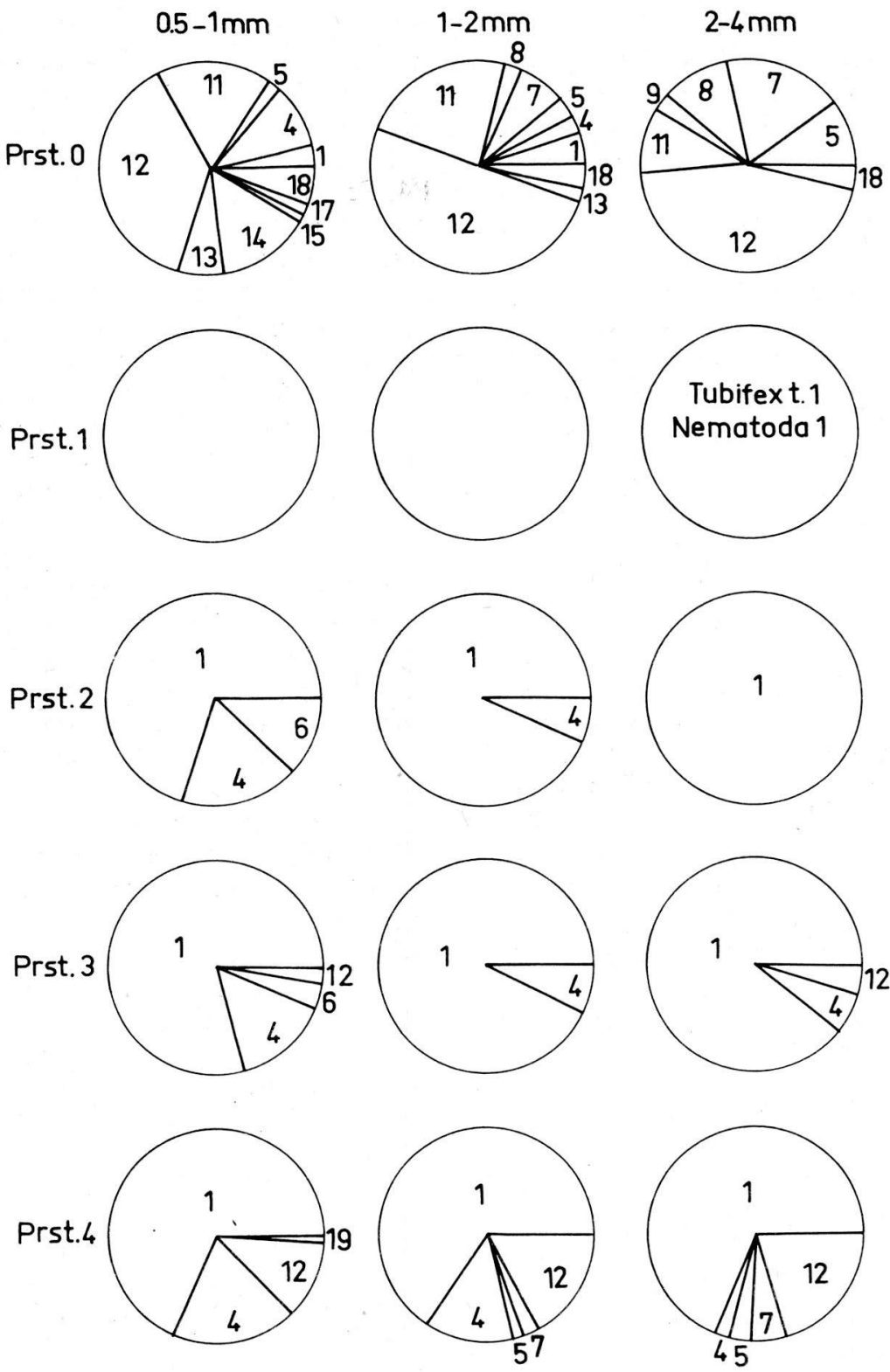

Abb. 8. Prozentuale Zusammensetzung der Tiergruppen an den einzelnen Probestellen bei verschiedenen Korngrößen. Die Zahl in dem Sektor gibt die Tiergruppe an. (Vergleiche die Legende zu Abb. 8a). 

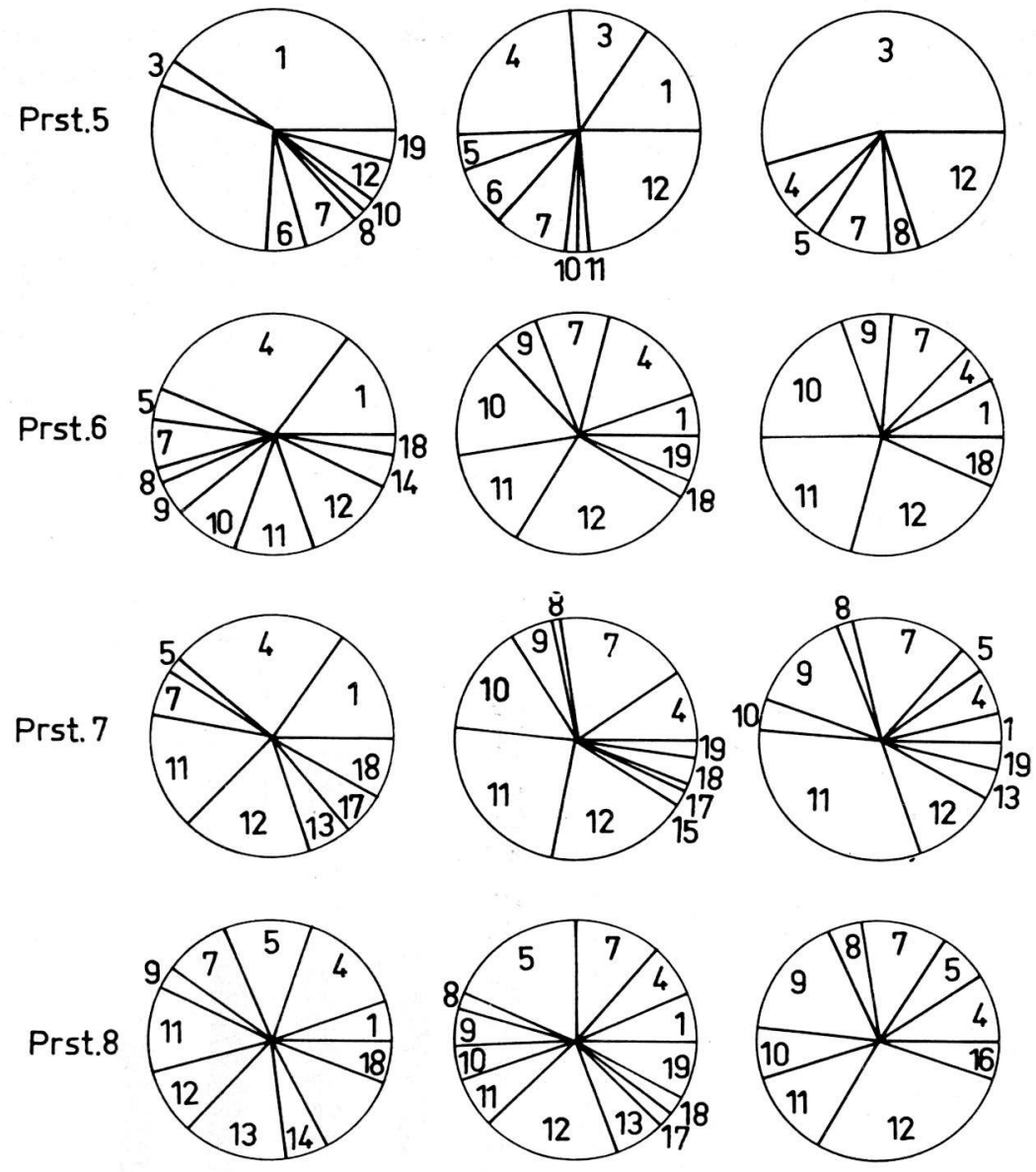

1 Tubificiden

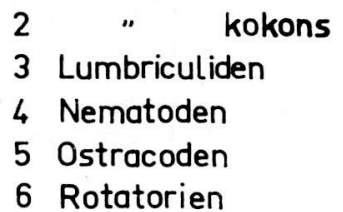

7 sonstige Insektenlarven / larvulae

8 Plecopterenlarven/Larvulae

9 Ephemeridenlarven 19 Chaetogaster

10 Turbellarien

11 Cyclopiden

12 Chironomiden

13 Hydrachnellen

14 Harpacticiden

15 Coelenteraten

16 Amphipoden

17 Milbennymphen

18 Naiden

Abb. 8a. Siehe Abb. 8. 
Interstitialschichten her. Die horizontale Einwanderung erscheint mir sehr unwahrscheinlich wegen der unterschiedlichen Korngrößenzusammensetzung der hyporheischen Sande (auf die Veröffentlichung der Resultate der Korngrößenzusammensetzung an den einzelnen Probenstellen muß hier verzichtet werden); wahrscheinlicher ist die Wiederbesiedlung der von mir untersuchten Tiefenbereiche des hyporheischen Interstitials von darunterliegenden Substratschichten her. Untersuchungen von Coleman und Hynes (1970), die im hyporheischen Interstitial noch in $70 \mathrm{~cm}$ Tiefe Tiere fanden, zeigten, daß nach Katastrophen (Hochwasser und Geschiebe) von hier aus eine Wiederbesiedlung erfolgte.

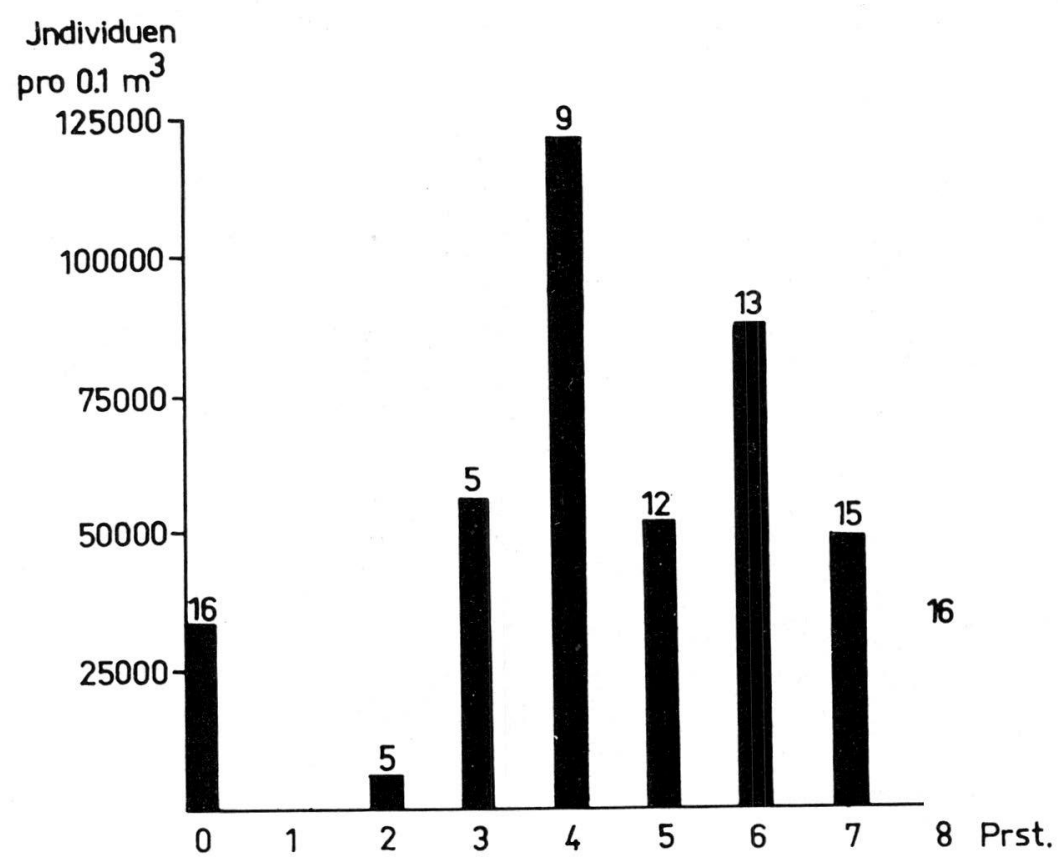

Abb. 9. Die absolute Besiedlungsdichte des hyporheischen Interstitials an den einzelnen Probestellen. Die Zahl über der Säule gibt die Anzahl der gefundenen Tiergruppen an. (Tubificidenkokons wurden zu Tubificiden gerechnet).

Die Probenstellen P7 und P8, sieht man von der Besiedlung durch Coelenteraten und Amphipoden ab, weisen eine deutliche Übereinstimmung mit der Besiedlung an P0 auf (Abb. 8 und 8a). Die tierische Besiedlung des hyporheischen Interstitials an P7 kann im Vergleich zu den Besiedlungsverhältnissen an P0 im wesentlichen als abgeschlossen betrachtet werden.

Die Besiedlungsdichte ist im Interstitial vom verfügbaren Porenraum und vom Angebot des aufnehmbaren Detritus abhängig. Die meisten Bewohner 
des Interstitials sind Detritusfresser, denn das hyporheische Interstitial ist als praktisch absoluter Dunkelraum ein in trophischer Hinsicht völlig vom Fließgewässer abhängiger Lebensraum. Die wenigen Räuber sind Turbellarien, Milben und einige Insektenlarven. So konnte Schwoerbel $(1961,1967)$ zeigen, daß für das kristalline Urgebirge eine direkte Proportionalität zwischen Besiedlungsdichte und Detritusmenge im hyporheischen Biotop besteht. Bei allochthoner Nährstoffzufuhr konnte in der Mettma jedoch eine eindeutige Proportionalität zwischen Besiedlungsdichte und dem Angebot an organischer Substanz resp. Detritus nicht festgestellt werden (Abb. 7 und 9). Es muss in diesem Zusammenhang noch darauf hingewiesen werden, daß mit der Bestimmung der organischen Substanz, noch nichts darüber ausgesagt ist, inwieweit die organische Substanz den Organismen als Energiequelle dienen, oder von ihnen in körpereigene Substanz umgesetzt werden kann (vgl. Wachs, 1967).

\section{DANKSAGUNG}

Danken möchte ich Herrn Prof. Dr. H. J. Elster für die zurverfügungstellung eines Arbeitsplatzes im Limnologischen Institut der Universität Freiburg (Falkau), Herrn Prof. Dr. J. Schwoerbel für wertvolle Hinweise und die Unterstützung meiner Untersuchungen, Frl. G. Tillmanns und Frl. G. Schulze für ihre technische Hilfe.

\section{ZUSAMMENFASSUNG}

Ein Mittelgebirgsbach (Mettma) erfährt eine punktförmige, allochthone Nährstoff- und Energiezufuhr durch häusliche und Brauereiabwässer. Der Untersuchungszeitraum erstreckte sich vom Mai 1970 bis Februar 1971. Die Abwassereinleitung führte zunächst zu einer generellen Verarmung und Umstrukturierung der Biozönose des hyporheischen Interstitials. Im Interstitial trat ein starkes $\mathrm{O}_{2}$-Defizit unmittelbar nach der Abwassereinleitung auf. Im Winter wies das hyporheische Interstitial höhere Temperaturen auf als die fliessende Welle. Bei allochthoner Nährstoffzufuhr konnte eine direkte Proportionalität zwischen dem Anteil an organischer Substanz und der Besiedlungsdichte nicht festgestellt werden. Die Besiedlungsdichte schwankt zwischen 0 und $1,2 \times 10^{5}$ Organismen pro $0,1 \mathrm{~m}^{3}$ Sediment. Nach $4,1 \mathrm{~km}$ zeigte die Biozönose in der tierischen Besiedlung eine ähnliche Zusammensetzung wie an der Bezugsprobenstelle P0. Nach 7,35 km Fließstrecke ist die Besiedlungsdichte geringfügig niedriger als an der Bezugsprobenstelle P0.

\section{LITERATUR}

ANGELIER, E. 1953. Recherches écologiques et biogéographiques sur la faune des sables submergés. Arch. zool. expér. gén., 90: 37-161.

CHAPPUIS, P. A. 1943. Über die Fauna des Spaltengewässers und des Grundwassers. Allantani Kozlemenyek, 40: 225-232.

COLEMAN, M. J. and HYNES 'H. E. N. 1970. The vertikal distribution of the invertebrate fauna in the bed of a stream. Limnol. oceanogr., 15: 31-40.

FRANKE, U. 1972. Hydrographie, Chemie und Nährstoffracht eines mit organischen Abwässern verunreinigten Gebirgsbaches. Arch. Hydrobiol. Suppl., 42: 95-124.

HUSMANN, S. 1956. Untersuchungen über die Grundwasserfauna zwischen Harz und Weser. Arch. Hydrobiol., 52: 1-184.

1966. Versuch einer ökologischen Gliederung des interstitiellen Grundwassers in Lebensbereiche eigener Prägung. Arch. Hydrobiol., 62: 231-268. 
LIEBMANN, H. 1960a. Handbuch der Frischwasser- und Abwasserbiologie, II. R. Oldenburg, München: 1-1149.

ORGHIDAN, TR. 1959. Ein neuer Lebensraum des unterirdischen Wassers: Der hyporheische Biotop. Arch. Hydrobiol., 55: 392-414.

REICHERT, W. und SIMON, M. 1972. Die Mettma - Ein Gebrigsbach als Brauereivorfluter. Mikrobiologische Untersuchungen entlang eines Abwasser-Substratgradienten. Arch. Hydrobiol. Suppl., 42: 125-138.

SCHWOERBEL, J. 1961. Über die Lebensbedingungen und die Besiedlung des hyporheischen Lebensraumes. Arch. Hydrobiol. Suppl., 25: 182-214.

1961a. Subterrane Wassermilben (Acari, Hydrachnellae, Porohalacaridae und Stygothrombiidae), ihre Ökologie und Bedeutung für die Abgrenzung eines aquatischen Lebensraumes zwischen Oberfläche und Grundwasser. Arch. Hydrobiol. Suppl., 25: 242306.

- 1967. Das hyporheische Interstitial als Grenzbiotop zwischen oberirdischem und subterranem Ökosystem und seine Bedeutung für die Primär-Evolution von Kleinsthöhlenbewohnern. Arch. Hydrobiol. Suppl., 33: 1-62.

- 1972. Produktionsbiologische Aspekte in Fließgewässern. Verhandlungsbericht der Deutschen Zoologischen Gesellschaft, 65. Jahresversammlung, Gustav Fischer Verlag: 57-64.

SOLOWIEW, M. M. 1924. Über die Rolle der Tubifex tubifex in der Schlammerzeugung. Int. Rev. Hydrobiol., 12: 90-101.

WACHS, B. 1967. Die Oligochaeten-Fauna der Fließgewässer unter besonderer Berücksichtigung der Beziehungen zwischen Tubificiden-Besiedlung und dem Substrat. Arch. Hydrobiol., 63: 310-386. 